\title{
La protection radiologique : objectifs et analyse économique
}

\author{
G. LACOURLY $(*)$
}

\section{(Manuscrit reçu le 23 avril 1975)}

Dans cet article, l'auteur tente d'analyser objectivement en quels termes le problème de la protection radiologique se pose pour les autorités nationales responsables de la santé publique.

\section{RÉSUMÉ}

La protection radiologique repose sur trois principes généraux : la limitation des doses individuelles, la justification de l'exposition, l'optimisation de la protection.

L'application pratique de ces principes conduit aux études suivantes :

- l'évaluation de l'exposition des individus du groupe critique,

- une analyse coût-avantage faisant apparaitre le bénéfice au regard des risques encourus,

- une analyse coût-efficacité mettant en évidence le niveau de protection optimal.

La première est devenue une étude classique, les deux autres relèvent des méthodes décisionnelles et soulèvent, à l'application, un certain nombre de difficultés, qui se situent plus dans le domaine de l'éthique que dans celui de la technique.

\begin{abstract}
Radiation protection - its purpose and economic appraisal.

There are three basic principles to radiation protection, viz the limitation of individual doses, justification of exposure and optimization of protection. The practical application of these principles requires the following investigations: an evaluation of the critical group exposure; a cost-benefit analysis showing the benefit versus specific hazards; a cost-effectiveness analysis demonstrating the optimal protection level. The first one is now a usual method, the other two belong to decision making methods and their application raises a number of difficulties in the ethical rather than in the technical field.
\end{abstract}

(*) Commissariat à l'Énergie Atomique, Département de Protection, B. P. nº 6, 92260 Fontenay-aux-Roses (France). 


\section{INTRODUCTION}

Le développement rapide de l'utilisation de l'énergie nucléaire auquel on assiste depuis quelques années, a eu pour corollaire l'intensification des recherches dans le domaine de la protection radiologique, aboutissant à une efficacité toujours plus grande des moyens mis en œuvre. Le résultat incontestable est que la protection de l'homme et de l'environnement contre les risques liés à l'industrie nucléaire atteint actuellement un niveau qui dépasse, et de très loin, celui de n'importe quelle autre industrie.

Avant d'aborder les principes généraux sur lesquels repose la protection radiologique, il faut noter tout d'abord que, parmi les êtres vivants, l'homme se trouve être le plus sensible aux rayonnements ionisants. Il en résulte que, bien que quelques organismes concentrent très fortement certains radionucléides prélevés dans le milieu, c'est encore la protection de l'homme qui est à la base de l'établissement des niveaux de protection dérivés fixés pour l'environnement. L'expérience montre, en effet, que ceux-ci restent très inférieurs aux concentrations nécessaires pour entraîner un effet biologique détectable sur la flore et la faune. Quant aux effets génétiques, pour lesquels on considère par prudence, mais sans preuve expérimentale, qu'il n'existe pas de seuil et que, par conséquent, toute dose, même minimale, peut entraîner un dommage, ils ne peuvent se distinguer de ceux qui sont dus à l'irradiation naturelle, dont les fluctuations d'un point à l'autre sont très supérieures aux doses additionnelles qui pourraient résulter des rejets de l'industrie nucléaire dans l'environnement. Au surplus, si l'on considère non plus les risques individuels, mais les risques pour la population d'une espèce dans son ensemble, les effets génétiques qui pourraient résulter de telles doses, seraient progressivement éliminés par le jeu de la sélection naturelle.

Finalement, on est donc fondé à affirmer que la protection radiologique de l'homme, telle qu'elle est actuellement conçue et assurée, entraîne ipso facto celle de l'environnement.

\section{OBJECTIFS DE LA PROTECTION RADIOLOGIQUE. PRINCIPES GÉNÉRAUX}

La protection radiologique du public repose sur le respect des limites de doses recommandées par la Commission Internationale de Protection Radiologique (CIPR) [1] et reprises par la plupart des réglementations nationales des pays parvenus au stade nucléaire.

En outre, ce principe de base conduisant à la limitation des expositions est complété par deux autres impératifs, qui découlent de l'hypothèse selon laquelle toute exposition même faible comporte un certain risque, à savoir :

- toute exposition comportant un certain risque, il importe que ce risque soit compensé par des avantages, dont l'importance justifie l'exposition; 
- il convient de maintenir toutes les doses aux valeurs les plus faibles auxquelles on peut parvenir raisonnablement, compte tenu des aspects sociaux et économiques.

En bref, la protection radiologique repose sur l'application de ces trois principes : le premier implique l'évaluation des doses individuelles délivrées au groupe de population le plus exposé, afin de les comparer aux limites de doses recommandées. Le second se traduit par une justification de l'exposition. Le troisième enfin conduit à la recherche d'une optimisation de la protection [2]. Ces deux derniers impératifs ne font plus appel à la notion de risque acceptable pour l'individu, mais à la notion de charge acceptable pour la société. Pour apprécier la charge pour la société, résultant du fonctionnement d'une installation, il faut alors faire appel à la notion de " détriment ", ellemême basée sur la notion de " dose collective ", qui tient compte à la fois des doses individuelles et du nombre de personnes exposées.

\section{LA LIMITATION DES DOSES INDIVIDUELLES}

Le principe de la limitation des doses individuelles, qui est à la base de l'établissement des normes de protection repose sur la notion de " risque acceptable pour l'individu ". Or, les risques résultant de l'exposition aux rayonnements sont des risques stochastiques, ce qui veut dire que lorsqu'un groupe de population est irradié, les effets n'apparaissent que chez quelques individus et ceci au hasard. C'est, en particulier, le cas des affections malignes, cancers et leucémies, et des effets génétiques. Or, dans les conditions actuelles, la preuve n'ayant pu être faite qu'il existe un seuil au-dessous duquel les effets des rayonnements ne produisent pas de dommage, force est bien d'établir les limites du risque individuel à une valeur que la société juge acceptable. C'est là un problème qui n'est pas particulier à la protection contre les rayonnements et qui se pose en termes analogues dans la plupart des activités humaines. On doit donc définir le niveau de risque individuel considéré comme acceptable. Les doses correspondantes constituent alors les limites individuelles, qui ne devront en aucun cas être dépassées. En règle générale, les dangers résultant de l'exposition aux rayonnements ionisants ne devraient pas dépasser ceux qui sont habituellement acceptés dans la vie courante. Le problème qui se pose, en pratique, dans chaque cas particulier, consiste à définir des mesures de protection propres à garantir que les limites de dose ne seront pas dépassées. Pour évaluer l'exposition des personnes du public résultant du rejet dans l'environnement de substances radioactives, on a, pendant un temps, fait appel à la notion de concentration maximale admissible dans l'air ou dans l'eau en admettant implicitement que l'exposition des individus était proportionnelle à la concentration des radionucléides dans le milieu où s'effectuait la dispersion initiale et on se contentait de s'assurer que cette concentration maximale était respectée. Mais, en raison de la complexité des voies qui peuvent conduire à une exposition de l'homme, cette approximation est souvent très insuffisante pour évaluer l'irradiation des individus du public. Une méthodologie s'est développée qui, mettant en évidence dans chaque cas les voies qui peuvent

voL. $10-\mathrm{N}^{\circ} 2$ 
conduire à une exposition de la population, permet d'évaluer de façon plus sûre l'irradiation correspondante. Elle tient compte de très nombreux facteurs, les uns caractérisant l'environnement, les autres caractérisant les populations. Il n'est, bien entendu, pas possible d'évaluer ainsi l'irradiation de chaque individu de la population, mais on peut généralement définir, au sein de celle-ci, des groupes de population composés d'individus ayant en ce qui concerne les facteurs qui conditionnent l'exposition (situation géographique, âge, habitudes alimentaires, habitudes professionnelles, mode de vie...) des caractéristiques semblables. A condition que les groupes de population ainsi définis soient suffisamment homogènes, il est raisonnable de considérer l'exposition moyenne à l'intérieur de chaque groupe comme représentative de l'exposition des individus qui le composent et c'est cette exposition moyenne que l'on comparera aux limites de dose pour les personnes du public. En pratique, les études menées dans chaque cas permettent d'établir l'existence d'un ou deux groupes de population dont les caractéristiques sont telles qu'ils seront soumis à une exposition plus élevée que le reste de la population et il suffira, pour garantir le respect des limites de dose, de s'assurer que l'exposition du ou des groupes critiques est inférieure à ces limites.

Il ne faut cependant pas oublier, dans cette analyse, que les limites de dose ne s'appliquent pas à l'irradiation résultant d'une source donnée, mais à l'irradiation totale subie par les personnes du public du fait de l'ensemble des sources, à l'exclusion toutefois de l'irradiation naturelle et de l'irradiation à des fins médicales. Dans le cas où plusieurs sources peuvent contribuer de façon significative à l'exposition, et ceci sera probablement de plus en plus fréquent dans l'avenir, la définition du groupe critique et l'évaluation de son irradiation devront tenir compte de ces différentes sources.

\section{LA JUSTIFICATION DE L'EXPOSITION}

Pour répondre à cette exigence, il convient de faire la preuve que le détriment additionnel causé par les rayonnements issus d'une source ou de plusieurs sources provenant d'une installation, trouve sa justification dans les avantages résultant du fonctionnement de cette installation. Pour ce faire, la méthode à mettre en œuvre entre dans le cadre de ce qu'on appelle aujourd'hui des "études décisionnelles " et appartient en l'occurence au type des " analyses coût-avantage ". termes :

Dans ce type d'analyse, le bilan se résume à la comparaison de deux

- les coûts comprennent toutes les dépenses d'investissement et de fonctionnement de l'installation, auxquelles viennent s'ajouter les coûts directs et indirects des conséquences des rejets radioactifs sur l'homme et son environnement,

- les avantages comprennent la valeur du produit de l'installation et tous les avantages directs et indirects qui en découlent pour l'ensemble de la population. 
Pour que l'exposition soit justifiée il faut que la valeur des avantages dépasse la valeur des coûts. Or nous avons vu que la valeur du "détriment » résultant du fonctionnement de l'installation est comptabilisée dans les coûts. L'expression du coût du détriment en termes monétaires est la source de difficultés qui seront examinées plus loin.

La justification de l'exposition est claire dans son principe, mais sur le plan moral elle semble $a$ priori soulever une difficulté car, de même que dans de nombreuses autres activités, le plus souvent le groupe de population qui subit le détriment n'est pas le même que celui qui reçoit les avantages. Cette jusification n'aurait donc aucun sens si les risques indivıduels n'étaient pas très petits. Le respect des limites de doses individuelles assurant une protection sûre contre tout risque significatif, il est cependant permis de considérer cette justification basée sur les résultats d'une analyse coût-avantage comme acceptable, même si les groupes de population concernés ne sont pas les mêmes.

\section{L'OPTIMISATION DE LA PROTECTION}

L'analyse coût-avantage permet de vérifier que l'exposition résultant de l'activité envisagée est justifiée. Il faut ensuite s'assurer que l'installation projetée fonctionne dans les meilleures conditions de protection, c'est-à-dire à un niveau tel que l'effort que nécessiterait une réduction supplémentaire de l'exposition ne serait pas justifié par la diminution du détriment correspondant. $\mathrm{Ce}$ but est atteint dans la pratique au moyen d'un calcul d'optimisation. Pour cela, on compare d'une part les avantages attendus d'une réduction de l'exposition, c'est-à-dire la valeur du détriment évité, et d'autre part le coût technologique des dispositifs nécessaires. A la différence de l'analyse coût-avantage, le bilan n'est plus global, mais se ramène à une évaluation différentielle des conséquences résultant du passage d'un niveau de protection à un autre. L'optimisation est obtenue en cherchant la valeur de l'exposition qui minimise la somme des deux termes. La solution optimale n'est toutefois acceptable qu'à la condition que les deux premiers principes soient respectés, c'est-à-dire que l'exposition des individus du groupe critique reste inférieure à la limite de dose fixée d'une part, et que l'exposition soit justifiée d'autre part.

\section{PROBLÈMES POSÉS PAR L'ÉVALUATION DU DÉTRIMENT}

Qu'il s'agisse de justifier ou d'optimiser l'exposition d'une population, il est nécessaire d'évaluer le détriment total, c'est-à-dire la somme des détriments individuels résultant de cette exposition. Pour y parvenir il faut tenir compte à la fois du risque individuel, c'est-à-dire de la probabilité d'occurence de l'effet nocif en fonction de la dose d'irradiation, de la gravité de cet effet et du nombre de personnes concernées.

Dans l'hypothèse où les différents risques sont liés à la dose reçue par des relations linéaires, le détriment individuel est directement proportionnel à la

voL. $10-\mathrm{N}^{\circ} 2$ 
dose. On peut alors évaluer le détriment total dans une population en multipliant la somme des doses reçues par les individus qui composent cette population par un coefficient de proportionnalité. La somme des doses individuelles, ou dose collective, peut également s'exprimer par le produit de la dose individuelle moyenne par le nombre d'individus de la collectivité étudiée.

La validité de l'utilisation du concept de dose collective, tel qu'il vient d'être défini, pour évaluer le détriment pour une population exposée repose sur la validité de l'hypothèse de la linéarité des relations entre la dose et les risques. Dans le cas contraire, en effet, le coefficient de proportionnalité entre la dose et le détriment ne serait pas constant et varierait avec la valeur de la dose et on devrait alors, pour apprécier le détriment pour la population, multiplier la fraction de la dose collective correspondant à chaque niveau de dose par un coefficient approprié. L'utilisation d'un coefficient de proportionnalité constant, déduit d'observations à dose élevée, conduirait alors à une surestimation du détriment, et celle-ci serait d'autant plus importante que les niveaux de dose seraient plus faibles.

L'évaluation du détriment soulève, en outre, une difficulté majeure, dès qu'on veut l'exprimer en termes monétaires. En effet, aussi bien dans l'analyse globale conduisant à la justification que dans l'analyse différentielle, conduisant à l'optimisation de la protection, les deux termes du bilan doivent être évalués dans la même unité. Les coûts étant exprimés en termes monétaires, le coût du détriment doit, lui aussi, s'exprimer dans la même unité. Un certain nombre d'estimation ont été faites de la valeur monétaire de la réduction de l'exposition de la population. Toutes tiennent compte explicitement ou implicitement de la valeur à accorder à la vie humaine. Il est évident qu'un tel problème ne peut être abordé qu'avec une extrême prudence.

Deux méthodes ont été employées. La première consiste à considérer l'homme comme un capital dans le processus de production économique et à estimer la valeur de ce capital par la valeur nette des biens qu'il est susceptible de produire par son travail au cours de sa vie active. Cette méthode fondée sur un aspect strictement économique des activités humaines présente l'avantage de la rigueur mathématique, mais ne tient aucun compte des aspects psychologiques du problème.

La seconde consiste à prendre pour base les dépenses consenties par la société pour sauver une vie humaine. Si ce calcul implicite de la valeur de la vie humaine peut paraître judicieux dans un certain nombre de domaines, l'utilisation a priori d'un tel résultat dans un calcul décisionnel dans un autre secteur peut apparaître comme très contestable. Les valeurs trouvées par ce biais peuvent, en effet, différer très largement suivant les situations et les objectifs poursuivis et le choix entre ces valeurs ne peut qu'être arbitraire. Il n'est pas sûr, en tout cas, que ce choix corresponde aux conditions du problème posé.

Finalement, l'évaluation du détriment en termes monétaires apparaît comme la difficulté majeure pour traiter les problèmes posés par la justification et l'optimisation et on ne peut établir de règle générale pour le choix de la méthode de quantification. 


\section{CONCLUSION}

Les principes fondamentaux sur lesquels est fondée la protection radiologique du public, permettent de s'assurer que les activités comportant une exposition aux rayonnements, en particulier dans le domaine des industries nucléaires, centrales électriques, usines de retraitement, aires de stockage de déchets radioactifs, sont exercées dans les meilleures conditions.

Sur le plan pratique, les méthodes permettant de garantir le respect des limites de dose individuelles, en particulier dans le cas de rejets de substances radioactives dans l'environnement, sont maintenant bien au point et une longue expérience a permis de s'assurer de la validité des modèles qui ont été développés pour l'évaluation de l'exposition des groupes critiques.

Quant à la justification et à l'optimisation de la protection, qui font appel aux théories décisionnelles, si le principe en est bien clair, leur application pratique soulève encore des problèmes qui ne peuvent être résolus qu'au moyen d'études poursuivies dans un cadre très élargi débordant le simple domaine de l'économétrie. A vrai dire, le problème de fond qui consiste à déterminer la charge acceptable pour la société en fonction des avantages apportés par l'activité développée n'est pas un problème propre à l'industrie nucléaire, non plus que l'évaluation du coût de la vie humaine. Aussi serait-il souhaitable que les procédures employées dans le domaine nucléaire, qui sont des procédures de pointe, soient étendues à la protection contre les autres risques avec une certaine unité de vue, ce qui contribuerait à résoudre, au moins en partie, les difficultés soulevées à l'occasion de l'évaluation du coût du détriment.

\section{BIBLIOGRAPHIE}

[1] Commission internationale de protection radiologique. Recommandations de la CIPR (adoptées le 17 septembre 1965). Publication CIPR-9.

[2] Commission internationale de protection radiologique. Les implications des recommandations de la Commission de maintenir les doses aux valeurs les plus faibles qu'il soit possible d'atteindre sans difficulté (rapport du Comité 4, 1973). Publication CIPR-22 Fontenay-aux-Roses, CEA, Département de Protection, 1974. 